\title{
Isotope pattern deconvolution as a successful alternative to calibration curve for application in wastewater-based epidemiology
}

\author{
Jorge Pitarch-Motellón ${ }^{1}$ • Lubertus Bijlsma ${ }^{1}$ - Juan Vicente Sancho Llopis ${ }^{1}$ • Antoni F. Roig-Navarro ${ }^{1}$ [D
}

Received: 28 December 2020 / Revised: 16 February 2021 / Accepted: 9 March 2021 / Published online: 17 March 2021

(C) Springer-Verlag GmbH Germany, part of Springer Nature 2021

\begin{abstract}
An isotope pattern deconvolution (IPD) quantification method has been applied for the determination of five substances (amphetamine, benzoylecgonine, cocaine, methamphetamine and MDMA) in wastewater for the application in wastewater-based epidemiology (WBE). A previously validated method that used a calibration curve for quantification was modified to apply IPD. The two approaches were compared in terms of analytical uncertainty in recovery studies of quality control samples, i.e. six wastewater samples from different geographical origins spiked at two concentration levels. Both methods were reliable as they passed $(z$-score $<2)$ in an interlaboratory exercise. After 60 individual determinations, IPD provided 11 results outside recovery limits (70-120\%) while the previous method produced 31 adverse results. All mean values for IPD were accurate whereas 6 out of 10 results showed RSD values higher than $30 \%$ or recoveries outside limits when using the former method. Moreover, the calculated method bias for the latter doubles that of IPD, which, in turn, makes the combined uncertainty $(u(c))$ much higher. Consequently, a simple change of data treatment-IPD quantification methodology - resulted in a lower uncertainty of the estimated illicit drug concentration, one of the main steps contributing to the final uncertainty in the normalized daily drug consumption through WBE. The current study demonstrated that the employment of IPD can also be very interesting for future applications of WBE, especially when matrix effects are high, complicating accurate quantification. In addition, when a high number of samples and/or compounds need to be analysed, IPD is faster than calibration and, eventually, cost-effective when isotopically labelled internal standard is highly expensive.
\end{abstract}

Keywords Wastewater analysis · Illicit drugs $\cdot$ Isotope dilution $\cdot$ Mass spectrometry $\cdot$ Isotope pattern deconvolution $\cdot$ Combined uncertainty

\section{Introduction}

Wastewater-based epidemiology (WBE) is an established approach to assess illicit drug consumption by a community and produces objective and near real-time data, which allows the monitoring of temporal and spatial trends [1-3]. It provides complementary information to traditional epidemiological drug use indicators based on general population surveys, data of hospitals and crime-related statistics $[1,4-6]$ and has been employed in several cities worldwide [2, 3, 7-9]. WBE is an emerging scientific research field to provide information on

Antoni F. Roig-Navarro

roig@uji.es

1 Research Institute for Pesticides and Water, Universitat Jaume I, Avda. Sos Baynat, s/n, 12071 Castelló, Spain the community's health and lifestyle habits, or environmental exposure [10]. Various new and potentially new applications are being developed to monitor exposure to pesticides [11], flame retardants [12] or bisphenol A [13]. A current hot topic is its potential as surveillance tool for disease outbreaks such as SARS-CoV-2 [14, 15]. The approach relies on a five-step strategy: (a) collection of representative 24-h composite influent wastewater samples, (b) concentration determination of human biomarkers in the samples, (c) calculation of daily loads, (d) normalization of daily loads to the population served by the wastewater treatment plant (WWTP) and (e) backcalculation to total daily consumption. Each of the steps is affected by different uncertainty sources [4, 16-18]. Drug biomarker chemical analysis represents a cornerstone within the WBE approach, since quantitative data are the basis of subsequent daily loads and back-calculations of drug use. Consequently, advanced analytical methodologies and 
expertise are required [19]. Moreover, uncertainty related to the concentration of drug biomarker in real samples is estimated as one of the main sources and depends, among others, on the analytical methodology and quantification strategy applied [18]. Thus, reducing the drug biomarker concentration uncertainty will have a major impact on the uncertainty of the total daily consumption [2]. A best-practice protocol for the analysis of illicit drug in wastewater has been suggested by the European Monitoring Centre for Drug and Drug Addiction (EMCDDA) [1] and formulated in the work of Van Nuijs et al. [9] where a multi-year inter-laboratory study allowed to improve analytical methodologies and the quality of reported data, thus helping to reduce the measurement uncertainty, an ever-searched goal in analytical chemistry.

Raw influent wastewater is one of the most complex environmental matrices, containing potentially thousands of interfering chemical substances. Reliable quantitative analytical methodologies for the determination of human biomarkers often at trace levels $(\mathrm{ng} / \mathrm{L})$ in such complex matrix are needed, but are challenging. Mass spectrometry (MS) is currently the method of choice for the determination of most human biomarkers in wastewater samples. However, well-known problems related with sensitivity, selectivity and precision, all of them affected by matrix effect, are to be faced. Since wastewater may vary strongly, and thus, each sample (i.e. matrix) can be potentially different; the matrix-matched calibration approach is not feasible. Therefore, high matrix effects are usually compensated by the addition of an isotopically labelled internal standard (ILIS), ideally a labelled standard of the target biomarker. This approach is referred to as isotope dilution mass spectrometry (IDMS) and is known to yield the most accurate, precise and reliable results [20].

The classical approach in WBE and MS-based methods involves the construction of a calibration curve in solvent in which the ratio of analyte/internal standard signals is plotted against the concentration of standard, with a constant amount of ILIS added to samples and calibrators. This procedure is able to compensate for losses during sample pretreatment, matrix effect or instrumental drift, but can also be costly when many compounds need to be analysed, i.e. ILIS of target compounds can be very expensive. In addition, the analysis, processing and integration of many standard lines can be timeconsuming. As an alternative quantification method to calibration curve IDMS, isotope pattern deconvolution (IPD) can be conducted. IPD is based on the alteration of the natural isotopologue abundances when a known amount of a labelled analogue of each analyte is added to a sample. For each substance, the isotopic abundances in the blend are a linear combination of those from the natural and from the labelled analogues. A deconvolution calculation based on multiple linear regression provides the concentration of the natural compound in each sample, i.e. each matrix [21-23]. Calculation is readily done with any spreadsheet software without the use of any methodological calibration, thus reducing the use of ILIS and the total analysis time. IPD calculation approach has been tested for the rapid and reliable quantifications of different compounds in several complex matrices, such as urine [24-27], serum [28], food [29] and environmental samples $[23,30-33]$. Recently, the power of IPD for the easy and accurate re-certification of vitamin D standards [34] has been demonstrated.

In this study, an IPD quantification method has been applied for the determination of five illicit drugs and/or metabolites in wastewater. The aims of the present work are to demonstrate the performance of IPD in terms of accuracy (trueness and precision) and to illustrate its potential and interest as alternative quantification methodology for future WBE applications. In addition, results are compared with data obtained from the application of an in-house method based on IDMS using a calibration curve for concentration calculation. The probable dispersion of results is calculated and compared in terms of within-lab reproducibility and combined uncertainty, $u(c)$. Furthermore, reliability of both methods is assessed by means of the participation in an international interlaboratory exercise. Finally, a rough estimation of the combined uncertainty to assess the whole overall uncertainty of illicit drug consumption through WBE model has been conducted.

\section{Materials and methods}

\section{Study summary}

Daily composite raw wastewater samples were collected over seven consecutive days from six different locations. The samples were routinely treated in the lab and analysed on different days. Quality controls (QCs) were prepared by spiking two sub-samples from each location at two concentration levels before sample treatment. Samples for QC preparation were selected among those collected in the middle of the week, where lower drug concentration was expected. After sample treatment, spiked concentrations in sample extracts were $2.5 \mu \mathrm{g} \mathrm{L}^{-1}$ (QC-L) and $20 \mu \mathrm{g} \mathrm{L}^{-1}$ (QC-H). Recoveries were calculated by subtracting the concentration found in the sample to the concentration in the corresponding QC (Eq. 1). Thus, six recovery values were obtained for each QC (six different wastewater compositions, i.e. matrices), allowing to estimate uncertainty with a sufficient level of confidence in intra-lab reproducibility conditions. A selection of 5 illicit drugs and/or metabolites was agreed in accordance with previous studies on WBE. Selected compounds for the present study were amphetamine (AMP), benzoylecgonine (BE), cocaine (COC), methamphetamine $(\mathrm{METH})$ and 3,4methylenedioxymethamphetamine (MDMA). 
Rec\% $=\frac{\text { Found QC Conc. }- \text { Found unspiked sample Conc. }}{\text { Spiked Conc. }} * 100$

Analysis was conducted by LC-(ID)MS/MS and concentrations calculated by conventional calibration curve (CAL) and isotope pattern deconvolution (IPD).

Afterwards, uncertainty was estimated from recovery values expressed as percentage according to a procedure based on the Nordtest Guide [35]. Parameters calculated were individual recovery for each compound and QC sample (Rec), mean recovery value for each compound of the six QCs, relative standard deviation of the mean value (RSD) and combined uncertainty $(u(c))$ taking into account all sources of bias (see the "Measurement uncertainty assessment" section below for a brief explanation, and Supplementary Information (ESM) for a detailed example of uncertainty calculation).

Accepted limits for recovery were 70-120\%. A limit of $30 \%$ (for comparison purposes) has been proposed for RSD and uncertainty in general. This limit is based on the Horwitz curve [36], where the expected within-laboratory reproducibility RSD is $30 \%$ for the $\mu \mathrm{g} \mathrm{L}^{-1}$ range of concentration.

A total of 30 individual results $(6$ spiked samples $\times 5$ compounds) and 5 mean results are obtained for each spiked concentration level (QC-L and QC-H). Previously to uncertainty assessment, outlier values were rejected using the Hampel test.

Furthermore, within the framework of the European collaborative research group SCORE, inter-laboratory studies have been organized to ensure high-quality analytical data in relation to the determination of illicit drug residues in wastewater used for WBE purposes [9]. Synthetic samples from this inter-laboratory comparison experiment, designed for the evaluation of the routine calibration curvebased method, were also determined by IPD and the results were sent for separate assessment. The inter-laboratory consisted in 3 tap water samples spiked with unknown levels of the target drugs (to be analysed three times each) and a control solution in $\mathrm{MeOH}$ (to be analysed five times). Accuracy of the method was assessed with $z$-score from the group's mean value.

\section{Chemicals and materials}

Certified reference materials of AMP, METH, MDMA, COC and $\mathrm{BE}$ as well as their corresponding isotope-labelled analogues AMP-d6, METH-d5, MDMA-d5, COC-d3 and BE-d3 were purchased from Cerilliant (Round Rock, TX, USA). All standards were acquired as solutions in methanol or acetonitrile.

Methanol (MeOH) HPLC, acetonitrile (ACN) HPLC, ammonium acetate and formic acid for LC-MS were acquired from Scharlau (Barcelona, Spain). Ultrapure water was obtained by purifying demineralised water $\left(\mathrm{H}_{2} \mathrm{O}\right)$ in a Milli$\mathrm{Q}$ plus system from Millipore (Bedford, MA, USA). Oasis HLB cartridges $\left(3 \mathrm{~cm}^{3}, 60 \mathrm{mg}\right)$ were from Waters (Milford, MA, USA).

Standard stock solutions of each non-labelled compound were prepared at $100 \mathrm{mg} \mathrm{L}^{-1}$ in $\mathrm{MeOH}$ or ACN. Stock solutions were used to prepare intermediate $10 \mathrm{mg} \mathrm{L}^{-1}$ solutions in $\mathrm{MeOH}$ or $\mathrm{ACN}$ and mixed to a $1 \mathrm{mg} \mathrm{L}^{-1}$ working solution in $\mathrm{MeOH}$ by diluting with the appropriate solvents. Individual stock solutions of isotope-labelled compounds were prepared in $\mathrm{MeOH}$ or $\mathrm{ACN}$ at $10 \mathrm{mg} \mathrm{L}^{-1}$. A surrogate mixed standard solution at $100 \mu \mathrm{g} \mathrm{L}^{-1}$ was prepared by volumetric dilution in $\mathrm{MeOH} / \mathrm{H}_{2} \mathrm{O} 1: 9 \mathrm{v} / \mathrm{v}$.

All standard solutions were stored in amber glass bottles at $-20{ }^{\circ} \mathrm{C}$.

Each working day, final calibration curves using mixed standards and surrogates were prepared by subsequent dilutions in $\mathrm{MeOH} / \mathrm{H}_{2} \mathrm{O}$ 1:9.

\section{Instrumentation}

Characterization and determination of analytes were performed on an Acquity UHPLC system from Waters (Milford, MA, USA) interfaced to a Xevo TQS triple quadrupole mass spectrometer from Waters (Manchester, UK) equipped with a T-Wave and electrospray ionization interface operated in positive mode (ESI+). Chromatographic separation was achieved using an Acquity UPLC BEH C18 column $(1.7 \mu \mathrm{m}, 50 \times 2.1 \mathrm{~mm})$, also from Waters, at a flow rate of $0.3 \mathrm{~mL} \mathrm{~min}{ }^{-1}$ and $40{ }^{\circ} \mathrm{C}$. Sample manager was kept at $5^{\circ} \mathrm{C}$. Mobile phase consisted of $5 \mathrm{mM}$ ammonium acetate and $0.01 \%$ formic acid in $\mathrm{H}_{2} \mathrm{O}$ (phase $\mathrm{A}$ ) and $\mathrm{MeOH}$ (phase $\mathrm{B}$ ). The gradient of phase $\mathrm{B}$ applied was as follows: initial conditions at $10 \%$, linear increase to $90 \%$ in $3 \mathrm{~min}, 90 \%$ (3$3.5 \mathrm{~min}$ ), $10 \%$ (3.6-6 $\mathrm{min}$ ) for equilibration of column.

Cone and desolvation gas (dry nitrogen) flows were set to 250 and $1200 \mathrm{~L} \mathrm{~h}^{-1}$ respectively. For operation in MS/MS mode, argon $99.995 \%$ (Praxair, Madrid, Spain) was used as collision gas, kept at $4 \cdot 10^{-3} \mathrm{mbar}$ and $0.15 \mathrm{~mL} / \mathrm{min}$ in the collision cell. Capillary voltage was $3.0 \mathrm{kV}$, source and desolvation temperatures were $150{ }^{\circ} \mathrm{C}$ and $650{ }^{\circ} \mathrm{C}$, respectively, and dwell times of $0.01 \mathrm{~s} /$ transition were selected.

All data were acquired using MassLynx v4.1 software (Waters, Manchester, UK) and processed using Masslynx and Microsoft's Excel spreadsheet software.

\section{Analytical procedure}

A slightly modified method previously developed and satisfactorily validated in the lab has been used in this study [37]. Briefly, $100 \mathrm{~mL}$ fourfold diluted influent wastewater samples were spiked with a mix of isotope-labelled analogues (approximately $100 \mu \mathrm{g} \mathrm{L}^{-1}$ of AMP-d6 and $10 \mu \mathrm{g} \mathrm{L}^{-1}$ for the other 
labelled compounds) and passed through previously conditioned Oasis HLB SPE cartridges. Analytes were eluted using $5 \mathrm{~mL}$ of $\mathrm{MeOH}$; extracts were evaporated to dryness and reconstituted in $1 \mathrm{~mL}$ of $\mathrm{MeOH} / \mathrm{H}_{2} \mathrm{O}$ 1:9 (v/v). A volume of $3 \mu \mathrm{L}$ of the final extracts was finally injected in the UHPLCMS/MS system. A calibration curve was prepared using 6 points plus blank in the range of 0.5 to $25 \mu \mathrm{g} \mathrm{L}{ }^{-1}$ and compared with IPD methodology (see the next section for a short explanation of IPD). The latter provides one result for each injected sample without the need of a calibration curve, avoiding as well processing and instrumental measurement steps. The mass spectrometry measurement of the analytes was adapted in order to apply IPD quantification (Table 1). The same sample extracts were quantified by both calculation methods and compared in terms of trueness, as percentage recovery of QCs, precision in terms of within-lab reproducibility RSD\% ( $\mathrm{n}=6)$, and accuracy assessed as $u(c)$.

\section{Quantification by isotope pattern deconvolution}

Traditionally, the peak area ratio between two given masses (or transitions in MS/MS) in the spiked sample is measured to build the calibration curve. However, when the isotopelabelled analogue is added, the resulting isotopic composition in the sample after the spike can be calculated as a linear combination of the natural and enriched compounds. Isotope pattern deconvolution (IPD) takes profit of this succeeding abundance in the blend, i.e. sample mixed with the labelled standard [21].

Table 1 The instrumental settings for MS/MS measurements needed for IPD

\begin{tabular}{|c|c|c|c|}
\hline Compound & MS/MS transitions & $\mathrm{CV}(\mathrm{V})$ & $\mathrm{CE}(\mathrm{V})$ \\
\hline AMP & $\begin{array}{l}136>119 \\
137>120\end{array}$ & 20 & 10 \\
\hline AMP-d6 & $\begin{array}{l}141>124 \\
142>125\end{array}$ & 20 & 10 \\
\hline $\mathrm{BE}$ & $\begin{array}{l}290>168 \\
291>169\end{array}$ & 40 & 20 \\
\hline BE-d3 & $\begin{array}{l}293>171 \\
294>172\end{array}$ & 40 & 20 \\
\hline $\mathrm{COC}$ & $\begin{array}{l}304>182 \\
305>183\end{array}$ & 30 & 20 \\
\hline COC-d3 & $\begin{array}{l}307>185 \\
308>186\end{array}$ & 30 & 20 \\
\hline METH & $\begin{array}{l}150>119 \\
151>120\end{array}$ & 40 & 40 \\
\hline METH-d5 & $\begin{array}{l}154>121 \\
155>121\end{array}$ & 40 & 40 \\
\hline MDMA & $\begin{array}{l}194>163 \\
195>164\end{array}$ & 30 & 15 \\
\hline MDMA-d5 & $\begin{array}{l}198>165 \\
199>165\end{array}$ & 30 & 15 \\
\hline
\end{tabular}

In brief, the total abundance in the mix for any transition in the mass spectrum $\left(A_{\mathrm{mix}}^{\mathrm{SRM} i}\right)$ can be calculated as a combination of two sources: the contribution of the natural compound (its abundance, $A_{\text {nat }}^{\mathrm{SRMi}}$, multiplied by its molar fraction, $X_{\text {nat }}$, in the blend) and that of the labelled compound $\left(A_{\text {lab }}^{\mathrm{SRMi}} X_{\text {lab }}\right)$ (Eq. 2).

$\left(A_{\text {mix }}^{\mathrm{SRMi}}\right)=\left(A_{\text {nat }}^{\mathrm{SRMi}}\right) X_{\text {nat }}+\left(A_{\text {lab }}^{\mathrm{SRMi}}\right) X_{\mathrm{i}}$

In the case of $n$ measured transitions in tandem MS, this can be expressed in matrix notation as follows (Eq. 3):

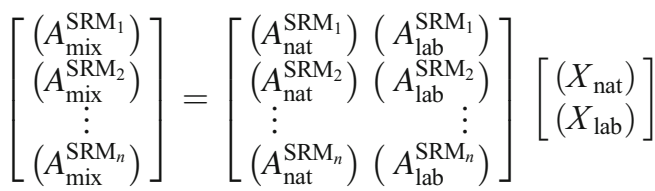

$$
\begin{aligned}
& +\left[\begin{array}{c}
e^{\mathrm{SRM}_{1}} \\
e^{\mathrm{SRM}_{2}} \\
\vdots \\
e^{\mathrm{SRM}_{n}}
\end{array}\right]
\end{aligned}
$$

where abundance values in the mix, $A_{\text {mix }}^{\mathrm{SRMi}}$, are experimentally measured. The matrix with the series of values $A_{\text {nat }}^{\mathrm{SRMi}}$ and $A_{\text {lab }}^{\mathrm{SRMi}}$ constitutes the reference abundance matrix and must be previously known.

In the IPD approach, the whole or part of the isotope pattern presented in the mix is measured in the MS/MS instrumental system. Usually, three or four transitions are measured. Knowing the distribution of abundances in the natural and labelled compounds, the corresponding molar fractions can be calculated by multiple linear regression. As we have more parameters (transitions) than unknowns (molar fractions), an error vector is included in Eq. 3. The best values of $X_{\text {nat }}$ and $X_{\mathrm{lab}}$ are found by least squares minimization of the error vector " $e$ ". This can be straightforwardly achieved with any spreadsheet software. (see ESM for a detailed IPD model and example of calculation explanation).

Finally, since the amount of labelled compound is known $\left(N_{\text {lab }}\right)$, the amount of natural compound in the sample $\left(N_{\text {nat }}\right)$ is readily obtained by Eq. 4:

$N_{\text {nat }}=N_{\text {lab }} \frac{X_{\text {nat }}}{X_{\text {lab }}}$

Hence, methodological calibration graph preparation and measuring are not needed. Quantification is directly conducted in the sample after one injection, leading to a cost and time savings. It requires, however, the full characterization of both compounds in terms of their isotopologue distribution of abundances and the exact concentration certification by reverse isotope dilution of the labelled compound standard solutions. Yet, this step only needs to be conducted once. Reference abundance of the equation matrix (Eq. 3) can be 
theoretically calculated or experimentally measured. In the present work, isotopologue abundances have been experimentally measured.

\section{Measurement uncertainty assessment}

Measurement uncertainty characterizes the dispersion of the values that could reasonably be attributed to a measurement result, $x \pm u(c)$. In analytical chemistry, uncertainty is usually reported as the standard deviation calculated from within-laboratory reproducibility experiments. However, if measurement uncertainty also takes into account bias, uncertainty can reach values which may be a factor of 2 to 5 times higher than previously. This fact simply reflects a much better estimation of the real variation in the measurement as, in this case, $u(c)$ represents all possible uncertainty sources [35]. For a method applied in a specific laboratory, this means within-lab precision, $u(\mathrm{RSD})$, and bias from a reference value, $u$ (bias) (Eq. 5). Estimation of this whole uncertainty makes the comparison of two results more suitably conducted. In the present work, $100 \%$ recovery is the reference value for bias uncertainty calculation. Correction factors have been used for the concentration of samples when QC recoveries are outside the accepted range. However, raw biases of QCs are employed to calculate $u$ (bias) as correction factors preclude bias calculation when using single-lab validation model from Nordtest Guide [35]. According to this guide for measurement uncertainty calculation, the sources of $u$ (bias) are the bias root mean square (RMS, which includes the difference with respect to the reference value and the reproducibility of this difference), and the uncertainty of the reference value itself, $u\left(C_{\mathrm{rec}}\right)$ (Eq. 6). The later one originated by the uncertainties associated with the concentration $(u(\mathrm{conc}))$ and quantity (volume, $u(\mathrm{vol}))$ of spiked standard (Eq. 7). $u$ (conc) is calculated from the uncertainty of the certified standard applying error propagation in dilution steps, and $u(\mathrm{vol})$ is calculated from the volumetric material tolerances in the lab (see the $\mathrm{u}(\mathrm{c})$ model tab in excel file provided in ESM for a detailed explanation).

$$
\begin{aligned}
& u(c)=\sqrt{u(\mathrm{RSD})^{2}+u(\text { bias })^{2}} \\
& u(\text { bias })=\sqrt{\mathrm{RMS}^{2}+u\left(C_{\mathrm{rec}}\right)^{2}} \\
& u\left(C_{\text {rec }}\right)=\sqrt{u(\text { conc })^{2}+u(\mathrm{vol})^{2}}
\end{aligned}
$$

\section{Results and discussion}

\section{Characterization of analytes}

Characterization of natural (AMP, BE, COC, METH and MDMA) and labelled compounds (AMP-d6,BE-d3,COC-d3,
METH-d5 and MDMA-d5) consisted in obtaining the reference matrix of isotopic abundances (Eq. 2) as well as the determination of the exact concentrations of ILIS solutions. Four transitions per compound, two from the natural and two from the labelled analogue clusters, were used for quantification purposes (Table 1).

Isotopic abundances were experimentally obtained taking into account the observed fragmentation pathways [38] and measurable transitions (around 10 transitions per compound). Suitable transitions were further selected beforehand by theoretical calculation of all possible transitions for each cluster, obtained using IsoPatrn software [39].

Concentrations of mixed labelled standard solutions (used for the spiking of samples) were certified by reverse isotope dilution (RID) analysis, which is based on the use of natural counterparts as reference for IPD calculations. Results of RID determinations were $535 \pm 21 \mu \mathrm{g} \mathrm{L}^{-1}$ for AMP-d6, $8.81 \pm 0.08 \mu \mathrm{g} \mathrm{L}^{-1}$ for BE$\mathrm{d} 3,10.75 \pm 0.08 \mu \mathrm{g} \mathrm{L}^{-1}$ for COC-d3, 17.14 $\pm 0.14 \mu \mathrm{g} \mathrm{L}^{-1}$ for METH-d5 and $12.81 \pm 0.10 \mu \mathrm{g} \mathrm{L}-1$ for MDMA-d5.

\section{Method performance comparison}

\section{Interlaboratory study}

Yearly inter-laboratory exercises are carried out with 37 participant laboratories from 25 countries [9]. As part of the validation and assessment of the calibration quantification method, the data set of IPD was also sent in 2017 to check the validity of the method. It is, however, noteworthy that the set was not included into the official testing calculations. Clearly, both methods passed the interlaboratory exercise $(z$-scores $<2)$ for the three spiked tap water samples and the methanol control solution (Table 2). This exercise is a test of the reliability of the methods. In general terms, IPD provided slightly higher concentrations than the CAL method, making it closer to group means for $\mathrm{BE}$ and equally well performing for AMP, while CAL results for MDMA and METH were nearer the group means. Variable results were found for $\mathrm{COC}$ with respect to group's mean.

\section{Recovery and RSD}

Comparison of both quantification approaches was carried out based on their performance in the determination of all 5 analytes in each QC samples. Here it is important to emphasize that the spiked wastewater samples were of different origins and that the matrix of each sample was non-identical. In fact, the composition can be very different as it can be affected daily by different weather conditions, industries, agriculture and population activities. Such conditions exemplify a strong test for the method accuracy. After the Hampel test for outlier detection, a total of 56 individual values (29 QC-L and 27 QCH) for IPD and 55 (26 QC-LL and 29 QC-H) for CAL were gathered. 


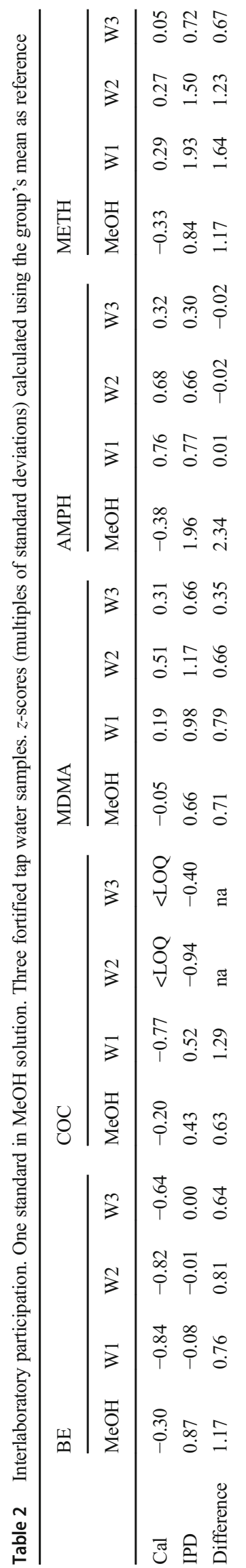

In general terms, IPD quantification provided higher recovery mean values for AMP, METH and MDMA, and lower for BE and COC than CAL (Table 3). Remarkably, IPD was able to correctly quantify $\mathrm{COC}$ and $\mathrm{BE}$, which have usually been found at high concentrations in the samples of the present study, and therefore, recovery experiments tend to be exceptionally difficult, especially when spiking low concentrations of standards (QC-L). This fact makes IPD a promising method, sensitive enough to accurately quantify low spiked concentrations in high blank and complex matrix samples. Taking into account the mean recovery values across the different samples, IPD showed more accurate determination of the analytes than those of CAL, where a great number of individual results were observed outside the accepted limits.

Following the accepted recovery results in typical validation studies, between 70 and $120 \%$, IPD calculation shows only $20 \%$ of individual recovery values (11 out of 56) outside the range in comparison to the $56 \%$ (31 out of 55) in the case of CAL. Being COC, with 9 out of 10 results outside the limits, the compound with poorer recoveries (Table 3). Taking into account that both quantification methods have been applied to the same extract, a reasonable explanation for the values outside these ranges is that matrix effect is not quantitatively compensated, being the IPD approach - based on abundances measurements - the less affected methodology.

In addition, no mean values were outside the accepted range for IPD while 6 (out of 10) fall beyond the limits for the CAL method. One more time COC shows the suboptimal recovery values.

Regarding precision, 2 (out of 10) RSD values higher than $30 \%$ were found for IPD whereas 6 (out of 10) fall outwards the limit for CAL method. Thus, IPD also showed a higher precision for the quantification, which makes the approach interesting and favourable for future applications.

\section{Combined uncertainty calculation and comparison}

Due to the high RSD values of method reproducibility observed, negligible effect of reference value uncertainty $u\left(C_{\text {rec }}\right)$ was expected on the final $u(c)$ value (see Eqs. 5-7). Method bias (RMS), however, is highly affected by recovery and RSD values and would show the main bias contribution to the combined uncertainty. Anyway, all uncertainty sources were calculated at each concentration level for each compound and sample. Table 4 summarizes results about the sources of uncertainty estimated for both quantification methods used.

As it was expected $u\left(C_{\mathrm{rec}}\right)$, the uncertainty associated with the reference value (100\% recovery) is very low $(11 \%)$. RMS (affected by the reproducibility of bias calculation) ranges from 9 to $81 \%$, with 2 (out of 10 ) values higher than $30 \%$ for IPD, and from 21 to $96 \%$, with 8 (out of 10) results higher than $30 \%$ for CAL. RMS stands for the highest contributor to $u$ (bias) (see Eq. 6), which in turn shows the same trends than RMS, as expected. 
Table 3 Recovery values for individual determinations and mean values at two concentration levels. For the mean values, RSD are also shown. In bold, those values are outside accepted limits (see text for a more detailed explanation)

\begin{tabular}{|c|c|c|c|c|c|c|c|c|c|c|c|}
\hline & & \multicolumn{10}{|c|}{ Recovery (\%) } \\
\hline & & \multicolumn{5}{|l|}{ IPD } & \multicolumn{5}{|l|}{ CAL } \\
\hline & & Amp & $\mathrm{BE}$ & $\mathrm{C}$ & MA & MDMA & Amp & $\mathrm{BE}$ & $\mathrm{C}$ & MA & MDMA \\
\hline 1-QC-L & & 141 & 72 & 116 & 107 & 115 & 101 & 148 & 172 & 54 & 72 \\
\hline 1-QC-H & & 87 & 70 & 105 & 110 & 108 & 42 & 111 & 157 & 53 & 69 \\
\hline 2-QC-L & & 94 & 120 & 89 & 115 & 95 & 108 & - & 204 & 76 & 104 \\
\hline 2-QC-H & & 99 & 85 & 110 & 109 & 109 & 69 & 85 & 165 & 84 & 108 \\
\hline 3-QC-L & & 51 & 80 & 107 & 105 & 118 & 78 & 143 & 190 & 44 & 78 \\
\hline 3-QC-H & & 116 & 78 & 109 & 105 & 112 & 64 & 137 & 184 & 56 & 80 \\
\hline 4-QC-L & & 112 & 33 & 102 & 114 & 106 & 76 & - & 108 & 112 & 100 \\
\hline 4-QC-H & & 126 & 73 & 113 & 117 & 115 & 92 & 124 & 193 & 95 & 102 \\
\hline 5-QC-L & & 31 & 24 & 86 & 129 & 100 & $\mathbf{0}$ & - & - & 72 & 99 \\
\hline 5-QC-H & & 95 & 62 & 110 & 114 & 104 & 94 & 14 & - & 128 & 84 \\
\hline 6-QC-L & & - & 265 & 148 & 135 & 120 & 36 & 76 & 148 & 48 & 68 \\
\hline 6-QC-H & & - & 93 & - & - & 116 & 43 & 165 & 150 & 52 & 69 \\
\hline \multirow[t]{2}{*}{ Mean (RSD) \% } & QC-L & $86(\mathbf{5 2})$ & 99 (89) & $108(21)$ & $117(10)$ & $109(9)$ & $67(62)$ & $122(33)$ & $164(23)$ & $68(37)$ & $87(18)$ \\
\hline & QC-H & $105(15)$ & $77(15)$ & $109(2)$ & $111(4)$ & $111(4)$ & $67(34)$ & 106 (49) & $170(11)$ & 78 (39) & 85 (19) \\
\hline
\end{tabular}

(-) eliminated value using Hampel test

QC-L, $2.5 \mu \mathrm{g} \mathrm{L}^{-1}$; QC-H, $20 \mu \mathrm{g} \mathrm{L}{ }^{-1}$

Finally, for all cases, $u(c)$ becomes significantly higher than RSD. A mean increase by a factor 2.5 was observed. However, as mean values for RSD and $u$ (bias) for IPD are significantly lower than those values for CAL, $u(c)$ for IPD is lower than $u(c)$ for CAL. Specifically, after $u(c)$ calculation, CAL methodology showed 9 of 10 results outside of $30 \%$ limit. On the other hand, for IPD, 4 of 10 results exceeded this reference value. Thus, $u(c)$ reinforces IPD as a more accurate quantification method, with higher trueness and precision.

In summary, IPD shows the higher trustworthy for all parameters associated with uncertainty. Calculation of $u(c)$ definitely shows that CAL can generate highly uncertain results, whereas IPD quantification methodology significantly decreases that combined uncertainty, which is always advantageous. Specifically, in the context of assessing the effectivity of implemented measures to reduce the impact of illicit drugs consumption, decreasing the uncertainty is essential. Some works dealing with illicit drug consumption estimation through wastewater analysis have tackled the problem of the associated uncertainty [16, 17]. A key paper by Castiglioni et al. [18] integrally addresses uncertainties associated with the estimation of community drug use through wastewater analysis using
Table 4 Combined, $u(c)$, and individual sources of uncertainty for IPD and CAL quantification methods. In bold, those values outside accepted limits. (See text for a more detailed explanation)

\begin{tabular}{|c|c|c|c|c|c|c|c|c|c|c|}
\hline \multirow{3}{*}{$\begin{array}{l}\text { Uncertainty } \\
\text { source }\end{array}$} & \multicolumn{10}{|c|}{ Uncertainty (\%) } \\
\hline & \multicolumn{5}{|l|}{ IPD } & \multicolumn{5}{|l|}{ CAL } \\
\hline & AMP & $\mathrm{BE}$ & $\mathrm{COC}$ & METH & MDMA & AMP & $\mathrm{BE}$ & $\mathrm{COC}$ & METH & MDMA \\
\hline$u(c)-\mathrm{L}$ & 66 & 121 & 32 & 25 & 19 & 80 & 114 & 71 & 56 & 29 \\
\hline$u(c)-\mathrm{H}$ & 23 & 31 & 14 & 16 & 16 & 52 & 70 & 67 & 54 & 30 \\
\hline RSD QC-L & 52 & 89 & 21 & 10 & 9 & 62 & 33 & 23 & 37 & 18 \\
\hline RSD QC-H & 15 & 15 & 2 & 4 & 4 & 34 & 49 & 11 & 39 & 19 \\
\hline$u$ (bias)-L & 40 & 81 & 24 & 23 & 17 & 51 & 96 & 67 & 41 & 22 \\
\hline$u$ (bias)-H & 17 & 27 & 14 & 15 & 16 & 40 & 49 & 66 & 37 & 23 \\
\hline RMS-L & 39 & 81 & 22 & 21 & 13 & 50 & 96 & 67 & 40 & 20 \\
\hline RMS-H & 14 & 25 & 9 & 11 & 12 & 39 & 48 & 65 & 36 & 21 \\
\hline$u\left(C_{\mathrm{rec}}\right)^{*}$ & \multicolumn{10}{|c|}{11} \\
\hline$u(\mathrm{vol})^{*}$ & \multicolumn{10}{|c|}{3} \\
\hline$u(\text { conc })^{*}$ & \multicolumn{10}{|c|}{10} \\
\hline
\end{tabular}

$* u$ (conc) and $u(\mathrm{vol})$ used to calculate $u\left(C_{\mathrm{rec}}\right)$. This one and RMS used to calculate $u$ (bias) (see Eqs. 5-7 and ESM for a detailed $u(c)$ model and example of calculation explanation) 
questionnaires, interlaboratory studies and already published research data. All critical steps were considered. The following RSD values are assigned to each step: $<10 \%$ for sampling $(u(S))$ and stability of drug biomarkers $(u(\mathrm{BS}))$; high variability $7-55 \%$ for the population size estimation $(u(\mathrm{PE}))$ in a catchment, where a case by case study is recommended; $1-34 \%$ associated with the replicated analysis of sewage drug biomarkers $(u(\mathrm{BA}))$ in sample; and back-calculation estimation of use step uncertainty ( $u(\mathrm{EU})$, available only for cocaine, used as example) shows a RSD of $26 \%$.

Thus, one more step to assess the whole uncertainty of illicit drug consumption through WBE model can be taken forward. Assuming that the different steps are not correlated, a combined uncertainty $(u(\mathrm{NDC}))$ for the final normalized daily drug consumption in a given population (mg/day/1000 people) can be estimated through error propagation (Eq. 8). As an example, common values from Castiglioni et al. [18] are used to assess the uncertainty for $\mathrm{COC}$ consumption estimation through the wastewater analysis of the local WWTP of Castelló (Spain). In that work, $23 \%$ is assigned to the population size uncertainty, $u(\mathrm{PE})$. Results from the present work are used to estimate the biomarker analysis uncertainty, $u(\mathrm{BA})$. The rest of the values (sampling, biomarker stability and estimation of use for cocaine) are directly taken up from the cited paper. Table 5 shows a comparison of $u(\mathrm{NDC})$ values for IPD and CAL quantification methods. The effect of using values of RSD (within-lab reproducibility) or $u(c)$ assessing the uncertainty associated with the biomarker concentration is also compared.

$u(\mathrm{NDC})=\sqrt{u(S)^{2}+u(\mathrm{BS})^{2}+u(\mathrm{BA})^{2}+u(\mathrm{EU})^{2}+u(\mathrm{EP})^{2}}(8$

RSD is the most widely used way to check measurement uncertainty in analytical chemistry. Using within-lab reproducibility obtained in the present work to estimate the uncertainty in biomarker analysis $(u(\mathrm{BA})=\mathrm{RSD})$, the calculated values for $u$ (NDC) fall around $40 \%$, no matter the quantification method used, IPD or CAL, and concentration level (Table 5).

Combined uncertainty, $u(c)$, is a less widely used although more rigorous and suitable way to evaluate measurement uncertainty. Using $u(c)$ to estimate $u(\mathrm{BA})$ provides similar results for $u(\mathrm{NDC})$ if IPD quantification method is applied.
Values for low and high concentration QCs are $49 \%$ and $40 \%$ respectively. However, if CAL quantification method is used, $u(\mathrm{NDC})$ nearly doubles its values, which ranges close to $80 \%$. This is in agreement with the higher $u(c)$ values for CAL method compared to IPD.

The recently established approach for monitoring illicit drug consumption in communities has several advantages. However, due to the different steps of the calculation model, the uncertainty of the last parameter $(u(\mathrm{NDC}))$ can be high as it has been roughly observed in the present work. One of the main steps contributing to the final uncertainty is the analysis of the drug biomarker. Although current analytical methodology is widely regarded as reliable, higher accuracy would always be welcome. In this way, a simple change of data treatment-IPD quantification methodology - has been shown as a route to explore. IPD has been shown, one more time, as a fast and reliable method. IPD results obtained are accurate with high trueness and precision, whereas no calibration curve (i.e. preparation, measurement and processing) is needed. Hence, one sample injection produces one analytical result, which leads, in consequence, to time savings.

The present work relates to the estimation of illicit drug consumption through wastewater analysis, however, IPD can be very interesting for future applications of WBE. As previously mentioned, WBE is an emerging scientific research field and is being implemented to obtain complementary information on the community's health and lifestyle habits, or environmental exposure $[10,15]$. In this context, IPD emerges as a promising quantification strategy due to the features that have been shown: reliable, fast and, eventually, cost-effective. This is particularly interesting when a high number of samples need to be analysed for many biomarkers.

\section{Conclusions}

In this work, an IPD quantification approach was applied for the first time to the determination of illicit drugs in wastewater-based epidemiology (WBE) studies. An IPD method was developed by modification of an already developed and validated calibration
Table 5 Combined uncertainty of the normalized daily cocaine consumption in Castelló (Spain) estimated for IPD and CAL quantification methods. RSD and $u(c)$ to calculate uncertainty associated with the biomarker analytical results, $u(\mathrm{BA})$, are compared. In bold, those values outside accepted limits. (See text for a more detailed explanation)

\begin{tabular}{|c|c|c|c|c|c|c|c|c|}
\hline & \multicolumn{4}{|l|}{ IPD } & \multicolumn{4}{|l|}{ CAL } \\
\hline & \multicolumn{2}{|l|}{ QC-L } & \multicolumn{2}{|l|}{ QC-H } & \multicolumn{2}{|l|}{ QC-L } & \multicolumn{2}{|l|}{ QC-H } \\
\hline & $u(\mathrm{BA})$ & $u(\mathrm{NDC})$ & $u(\mathrm{BA})$ & $u(\mathrm{NDC})$ & $u(\mathrm{BA})$ & $u(\mathrm{NDC})$ & $u(\mathrm{BA})$ & $u(\mathrm{NDC})$ \\
\hline$u(\mathrm{BA})=u(c)$ & 32 & 49 & 14 & 40 & 71 & 80 & 67 & 77 \\
\hline$u(\mathrm{BA})=\mathrm{RSD}$ & 21 & 43 & 2 & 38 & 23 & 44 & 11 & 39 \\
\hline
\end{tabular}

$u(c)$ and RSD values used to assess $u(\mathrm{BA})$ values are taken from Table 4 
curve-based isotope dilution mass spectrometry methodology. Amphetamine, benzoylecgonine, cocaine, methamphetamine and MDMA were selected as target analytes. A rough study of uncertainty was carried out for both quantification methods based on the performance in quality control analysis of routine WBE samples in 6 batches from different locations.

Reliability of the methods was successfully checked through an international inter-laboratory study with $z$-scores $<2$ for both methods.

The proposed IPD methodology consistently showed better trueness results in terms of recovery percentage in QC analysis. The application of IPD quantification on the same QC sample extracts allowed to reduce the number of out-of-range recovery results (70-120\%) from 31 to 11 out of 60 total individual values, compared to calibration-based quantification. Although the presence of analytes on the samples employed to QC preparation produced additional difficulty in recovery studies, the average recovery percentages at high and low concentrations showed acceptable results between 70 and $120 \%$ for all compounds in the case of IPD. By contrast, most calibration average recoveries (6 out of 10) fell outside that range.

On the other hand, recovery reproducibility and bias are significantly better for IPD quantification method, being bias reproducibility (RMS) the main factor affecting bias. As a general trend, values for IPD methodology are a half those for CAL. The later shows 8 out of 10 results higher than the proposed limit of $30 \%$.

As a consequence of the above, combined uncertainties $(u(c))$ for IPD were considerably lower than those for calibration. This, in turn, resulted in lower uncertainty of normalized daily drug consumption (NDC), which is one of the main parameters of interest in WBE.

IPD can be, therefore, very interesting for future applications of wastewater-based epidemiology, especially when high matrix effects hamper an accurate quantification. IPD is more accurate, faster and eventually cost-effective than calibration methods. It has been shown as an alternative to calibration-based methods, including matrix-matched calibration, and a route to explore for future applications.

Supplementary Information The online version contains supplementary material available at https://doi.org/10.1007/s00216-021-03287-7.

Acknowledgements The authors acknowledge C. Ort from EAWAG $(\mathrm{CH})$ for his invaluable comments to improve the manuscript.

Author contributions J. Pitarch-Motellón: methodology, investigation, writing - reviewing and editing, visualization

L. Bijlsma: methodology, investigation, writing-reviewing and editing

J.V. Sancho Llopis: methodology, writing - reviewing and editing

A. F. Roig-Navarro: conceptualization, formal analysis, writingoriginal draft
Funding This work received financial support from Generalitat Valenciana, Spain, as research group of excellence (PROMETEO II/2014/023).

\section{Declarations}

Conflict of interest The authors declare no competing interests.

\section{References}

1. European Monitoring Centre for Drugs and Drug Addiction. Assessing Illicit Drugs in Wastewater: Advances in Wastewaterbased Drug Epidemiology, Insights 22. Luxembourg: Publications Office of the European Union; 2016.

2. Ort C, van Nuijs ALN, Berset JD, Bijlsma L, Castiglioni S, Covaci A, et al. Spatial differences and temporal changes in illicit drug use in Europe quantified by wastewater analysis. Addiction. 2014;109: 1338-52. https://doi.org/10.1111/add.12570.

3. González-Mariño I, Baz-Lomba JA, Alygizakis NA, Andrés-Costa MJ, Bade R, Bannwarth A, et al. Spatio-temporal assessment of illicit drug use at large scale: evidence from 7 years of international wastewater monitoring. Addiction. 2020;115:109-20. https://doi. org/10.1111/add.14767.

4. Zuccato E, Chiabrando C, Castiglioni S, Bagnati R, Fanelli R. Estimating community drug abuse by wastewater analysis. Environ Health Perspect. 2008;116:1027-32. https://doi.org/10. 1289/ehp.11022.

5. Banta-Green C, Field J. City-wide drug testing using municipal wastewater. A new tool for drug epidemiology. Significance. 2011;8:70-4. https://doi.org/10.1111/j.1740-9713.2011.00489.x.

6. van Nuijs ALN, Castiglioni S, Tarcomnicu I, Postigo C, Lopez de Alda $\mathrm{M}$, Neels $\mathrm{H}$, et al. Illicit drug consumption estimations derived from wastewater analysis: a critical review. Sci Total Environ. 2011;409:3564-77. https://doi.org/10.1016/j.scitotenv.2010.05.030.

7. van Nuijs ALN, Mougel JF, Tarcomnicu I, Bervoets L, Blust R, Jorens PG, et al. Sewage epidemiology - a real-time approach to estimate the consumption of illicit drugs in Brussels, Belgium. Environ Int. 2011;37:612-21. https://doi.org/10.1016/j.envint.2010.12.006.

8. Thomas KV, Bijlsma L, Castiglioni S, Covaci A, Emke E, Grabic $\mathrm{R}$, et al. Comparing illicit drug use in 19 European cities through sewage analysis. Sci Total Environ. 2012;432:432-9. https://doi. org/10.1016/j.scitotenv.2012.06.069.

9. van Nuijs ALN, Lai FY, Been F, Andres-Costa MJ, Barron L, BazLomba JA, et al. Multi-year inter-laboratory exercises for the analysis of illicit drugs and metabolites in wastewater: development of a quality control system. TrAC - Trends Anal Chem. 2018;103:34 43. https://doi.org/10.1016/j.trac.2018.03.009.

10. Lorenzo M, Picó Y. Wastewater-based epidemiology: current status and future prospects. Curr Opin Environ Sci Health. 2019;9:77-84. https://doi.org/10.1016/j.coesh.2019.05.007.

11. Rousis NI, Zuccato E, Castiglioni S. Monitoring population exposure to pesticides based on liquid chromatography-tandem mass spectrometry measurement of their urinary metabolites in urban wastewater: a novel biomonitoring approach. Sci Total Environ. 2016;571: 1349-57. https://doi.org/10.1016/j.scitotenv.2016.07.036.

12. Been F, Bastiaensen M, Lai FY, van Nuijs ALN, Covaci A. Liquid chromatography-tandem mass spectrometry analysis of biomarkers of exposure to phosphorus flame retardants in wastewater to monitor community-wide exposure. Anal Chem. 2017;89:10045-53. https://doi.org/10.1021/acs.analchem.7b02705.

13. Lopardo L, Petrie B, Proctor K, Youdan J, Barden R, KasprzykHordern B. Estimation of community-wide exposure to bisphenol A via water fingerprinting. Environ Int. 2019;125:1-8. https://doi. org/10.1016/j.envint.2018.12.048. 
14. Medema G, Heijnen L, Elsinga G, Italiaander R, Brouwer A. Presence of SARS-Coronavirus-2 in sewage. https://doi.org/10. 1101/2020.03.29.20045880

15. Sims N, Kasprzyk-Hordern B. Future perspectives of wastewaterbased epidemiology: monitoring infectious disease spread and resistance to the community level. Environ Int. 2020;139:105689. https://doi.org/10.1016/j.envint.2020.105689.

16. Mathieu C, Rieckermann J, Berset JD, Schürch S, Brenneisen R. Assessment of total uncertainty in cocaine and benzoylecgonine wastewater load measurements. Water Res. 2011;45:6650-60. https://doi.org/10.1016/j.watres.2011.09.049.

17. Lai FY, Ort C, Gartner C, Carter S, Prichard J, Kirkbride P, et al. Refining the estimation of illicit drug consumptions from wastewater analysis: co-analysis of prescription pharmaceuticals and uncertainty assessment. Water Res. 2011;45:4437-48. https://doi.org/10. 1016/j.watres.2011.05.042.

18. Castiglioni S, Bijlsma L, Covaci A, Emke E, Hernández F, Reid M, et al. Evaluation of uncertainties associated with the determination of community drug use through the measurement of sewage drug biomarkers. Environ Sci Technol. 2013;47:1452-60. https://doi. org/10.1021/es302722f.

19. Hernández F, Castiglioni S, Covaci A, de Voogt P, Emke E, Kasprzyk-Hordern B, et al. Mass spectrometric strategies for the investigation of biomarkers of illicit drug use in wastewater. Mass Spectrom Rev. 2018;37:258-80. https://doi.org/10.1002/mas.21525.

20. Furey A, Moriarty M, Bane V, Kinsella B, Lehane M. Ion suppression; a critical review on causes, evaluation, prevention and applications. Talanta. 2013;115:104-22. https://doi.org/10.1016/j. talanta.2013.03.048

21. García-Alonso JI, Rodríguez-González P. Isotope dilution mass spectrometry. R Soc Chem. 2013.

22. González-Antuña A, Rodríguez-González P, Centineo G, García Alonso JI. Evaluation of minimal 13C-labelling for stable isotope dilution in organic analysis. Analyst. 2010;135:953. https://doi.org/ 10.1039/b924432h.

23. Castillo Á, Gracia-Lor E, Roig-Navarro AF, Vicente Sancho J, Rodríguez-González P, Alonso JIG. Isotope pattern deconvolution-tandem mass spectrometry for the determination and confirmation of diclofenac in wastewaters. Anal Chim Acta. 2013;765:77-85. https://doi.org/10.1016/j.aca.2012.11.033.

24. González-Antuña A, Rodríguez-González P, Centineo G, García Alonso JI. Simultaneous determination of seven $\beta 2$-agonists in human and bovine urine by isotope dilution liquid chromatography-tandem mass spectrometry using compound- specific minimally 13C-labelled analogues. J Chromatogr A. 2014;1372:63-71. https://doi.org/10.1016/j.chroma.2014.10.065.

25. Pitarch-Motellón J, Roig-Navarro AF, Sancho JV, Ibáñez M, Fabregat-Cabello N, Pozo OJ, et al. Evaluation of uncertainty sources in the determination of testosterone in urine by calibration-based and isotope dilution quantification using ultra high performance liquid chromatography tandem mass spectrometry. J Chromatogr A. 2017;1508:73-80. https://doi.org/10.1016/j. chroma.2017.05.072.

26. Pitarch-Motellón J, Sancho JV, Ibáñez M, Pozo O, Roig-Navarro AF. Determination of selected endogenous anabolic androgenic steroids and ratios in urine by ultra high performance liquid chromatography tandem mass spectrometry and isotope pattern deconvolution. J Chromatogr A. 2017;1515:172-8. https://doi. org/10.1016/j.chroma.2017.08.006.

27. Ereño Artabe A, González-Gago A, Suarez Fernández A, Pitarch Motellón J, Roig-Navarro AF, Pozo OJ, et al. Isotope dilution LCESI-MS/MS and low resolution selected reaction monitoring as atool for the accurate quantification of urinary testosterone. $\mathrm{J}$ Pharm Biomed Anal. 2019;163:113-21. https://doi.org/10.1016/j. jpba.2018.09.038.

28. Pitarch-Motellón J, Fabregat-Cabello N, Le Goff C, Roig-Navarro AF, Sancho-Llopis JV, Cavalier E. Comparison of isotope pattern deconvolution and calibration curve quantification methods for the determination of estrone and $17 \beta$-estradiol in human serum. J Pharm Biomed Anal. 2019;171:164-70. https://doi.org/10.1016/j. jpba.2019.04.013.

29. Fabregat-Cabello N, Zomer P, Sancho JV, Roig-Navarro AF, Mol HGJ. Comparison of approaches to deal with matrix effects in LCMS/MS based determinations of mycotoxins in food and feed. World Mycotoxin J. 2016;9:149-61. https://doi.org/10.3920/ WMJ2014.1872.

30. Fabregat-Cabello N, Pitarch-Motellón J, Sancho JV, Ibáñez M, Roig-Navarro AF. Method development and validation for the determination of selected endocrine disrupting compounds by liquid chromatography mass spectrometry and isotope pattern deconvolution in water samples. Comparison of two extraction techniques. Anal Methods. 2016;8:2895-903. https://doi.org/10. 1039/C6AY00221H.

31. Fabregat-Cabello N, Sancho JV, Vidal A, González FV, RoigNavarro AF. Development and validation of a liquid chromatography isotope dilution mass spectrometry method for the reliable quantification of alkylphenols in environmental water samples by isotope pattern deconvolution. J Chromatogr A. 2014;1328:43-51. https://doi.org/10.1016/j.chroma.2013.12.077.

32. Castillo A, Rodríguez-González P, Centineo G, Roig-Navarro AF, Alonso JIG. Multiple spiking species-specific isotope dilution analysis by molecular mass spectrometry: simultaneous determination of inorganic mercury and methylmercury in fish tissues. Anal Chem. 2010;82:2773-83. https://doi.org/10.1021/ac9027033.

33. Fabregat N, Rodriguez-Gonzalez P, Castillo A, Malherbe J, Navarro AFR, Long S, et al. Fast and accurate procedure for the determination of $\mathrm{Cr}(\mathrm{VI})$ in solid samples by isotope dilution mass spectrometry. Environ Sci Technol. 2012;46:12542-9. https://doi. org/10.1021/es3022864.

34. Pitarch-Motellón J, Fabregat-Cabello N, Le Goff C, Roig-Navarro AF, Cavalier E. Re-certification of hydroxyvitamin D standards by isotope pattern deconvolution. J Chromatogr B. 2019;1120:89-94. https://doi.org/10.1016/j.jchromb.2019.04.043.

35. Magnusson B, Näykki T, Hovind H, Krysell M. Handbook for calculation of measurement uncertainty in environmental laboratories. Nordtest technical report 537, ed. 3. Nordtest, 2011.

36. Albert R, Horwitz W. A heuristic derivation of the Horwitz curve. Anal Chem. 1997;69:789-90. https://doi.org/10.1021/ac9608376.

37. Bijlsma L, Beltrán E, Boix C, Sancho JV, Hernández F. Improvements in analytical methodology for the determination of frequently consumed illicit drugs in urban wastewater. Anal Bioanal Chem. 2014;406:4261-72. https://doi.org/10.1007/ s00216-014-7818-4.

38. Bijlsma L, Sancho JV, Hernández F, Niessen WMA. Fragmentation pathways of drugs of abuse and their metabolites based on QTOF MS/MS and $\mathrm{MS}^{\mathrm{E}}$ accurate-mass spectra. J Mass Spectrom. 2011;46:865-75. https://doi.org/10.1002/jms.196.

39. Ramaley L, Herrera LC. Software for the calculation of isotope patterns in tandem mass spectrometry. Rapid Commun Mass Spectrom. 2008;22:2707-14. https://doi.org/10.1002/rcm.3668.

Publisher's note Springer Nature remains neutral with regard to jurisdictional claims in published maps and institutional affiliations. 